\title{
What Do Residents Want from Wellness? A Needs Assessment of Psychiatry Residents to Inform a Residency Wellness Strategy
}

\author{
Yasmin Nasirzadeh $^{1} \cdot$ Laura Chertkow $^{1} \cdot$ Sarah Smith $^{1} \cdot$ Certina Ho $^{1} \cdot$ Mark Fefergrad $^{1} \cdot$ Deanna Chaukos $^{1} \oplus$
}

Received: 27 June 2021 / Accepted: 6 December 2021 / Published online: 22 January 2022

(c) Academic Psychiatry 2021

Wellness initiatives in postgraduate education are increasingly being recognized as essential and prioritized by programs $[1,2]$. There is an important link between physician wellbeing and patient outcomes [3, 4]. The postgraduate learning environment is complex and is a major contributor to resident burnout. Common stressors for residents include navigating wide responsibility and expertise gaps, at times working with little support from supervisors, juggling personal and professional demands, lack of control over their time, and navigating challenges inherent to medicine [5]. These stressors, though common to all physicians, are exacerbated for trainees [6].

Recognition of the impact of the learning environment on resident wellbeing has led to widespread adoption of wellness programming. There is growing experience among diverse residency programs with different types of wellness supports, from curricular content to mental health care access and referral pathways [7, 8]. However, there is little evidence to support stand-alone interventions, and little guidance on how to develop a wellness strategy [9]. Wellness programs that fall short can have the opposite effect-they can invalidate wellbeing concerns and disengage residents. Further, wellness can be supported at different levels-individual, community, and systems [10]. It is important that residency programs undertake a needs assessment of their unique learning environment to inform a wellness strategy.

Here we describe a 3-year initiative (2018-2020) to develop a wellness strategy in the University of Toronto Psychiatry Residency Program. We describe the development of a wellness subcommittee integrated as part of the Residency Program Committee (RPC) organization chart, the use of a multi-pronged needs assessment to define our goals, which then informed the mission for our Wellbeing

Deanna Chaukos

Deanna.Chaukos@sinaihealth.ca

1 University of Toronto Temerty Faculty of Medicine, Toronto, ON, Canada subcommittee and early priorities for initiatives. According to the American Psychiatric Association (APA) Toolkit for Wellbeing Ambassadors framework [11], there are seven key aspects of an evidence-based wellness strategy, including (1) educate and increase awareness, (2) designate time for reflection, (3) teach practical skills, (4) build community, (5) ensure access to care, (6) improve workplace environment, and (7) transform institutional culture. We used this framework to guide our work.

\section{Description of the Needs Assessment Methods}

With support of the Program Director and the RPC (the residency's primary governing body that oversees postgraduate Psychiatry training), a subcommittee of the RPC was developed to support wellness initiatives in our residency. Our RPC Wellbeing Subcommittee included a new faculty Wellness Lead, invested faculty representing diverse areas of residency education (International Medical Graduate education, Faculty Development, and representatives from the Postgraduate Wellness Office), and two resident leads (elected representatives of the resident-led Wellness Committee [RWC]). This group pursued a multi-pronged needs assessment to define goals for the new RPC Wellbeing Subcommittee.

The needs assessment included two components: a residency-wide Town Hall meeting emphasizing a qualitative approach, which utilized individual written reflections and a group-facilitated World Café Model [12], as well as a subsequent residency-wide survey. Our study was formally exempt from University of Toronto Research Ethics Board review. All residents were invited to the Town Hall through programmatic email communication. Teaching-faculty with interest/expertise in wellness were invited to join. Sixteen general psychiatry residents out of $187(\sim 9 \%)$ and three faculty members attended the Town Hall. Before the Town Hall began, resident and 
faculty participants completed a brief written reflection, which prompted participants to define physician wellbeing and factors that influence their wellbeing. A World Café Model was used to facilitate the Town Hall discussion: participants rotated through three small-group discussions, each with a facilitator to encourage participation and take notes. Small-groups explored current strengths and gaps in wellness offerings and discussed priority areas for next steps in programming, which were later discussed as a large group.

Following the Town Hall meeting, the RWC resident representatives and the faculty Wellness Lead used an inductive thematic analysis to interpret the data from the individual reflections and notes from the small-groups. We identified prominent themes and points to clarify, which guided development of a focused follow-up survey for all Psychiatry residents. The electronic survey aimed to elucidate if there was widespread interest in programmatic suggestions generated at the town hall. One hundred and ten of 187 residents (59\%) completed the survey, with balanced representation of residents from across PGY1-5 years (20\% PGY1, 17\% PGY2, 21\% PGY3, 23\% PGY4, 19\% PGY5). In addition to multiple choice questions aimed at querying interest in diverse wellness offerings, the survey also allowed for four free-text responses.

The RWC leads and the faculty Wellness Lead evaluated all descriptive needs assessment data, including handwritten responses from the Town Hall reflections, facilitator notes from the World Café Model small-groups, and free-text responses from the survey. An inductive approach was used to identify common themes, and a deductive approach integrated knowledge of the resident wellness literature and the APA Ambassadors Toolkit to draw comparison. Each individual interpreted the data independently; then, inferences drawn were discussed as a group. The results of this analysis, corroborated by the quantitative results of the survey, informed three guiding principles of the residency program's wellness strategy (see "Results of the Needs Assessment").

The needs assessment data, in addition to the guiding principles, were revisited annually utilizing a constant comparison method [13] by the new resident leads of the RWC and the same Faculty Wellness Lead, with the aim of describing yearly goals of the RPC Wellbeing Subcommittee. Similar themes were noticed upon re-evaluation of the needs assessment yearly; however, contextual events in the residency occurring at the time allowed for new interpretations and areas of focus.

\section{Results of the Needs Assessment}

Firstly, we learned that one size does not fit all; junior (PGY1-2) and senior residents (PGY3-5) identified different wellness needs. For example, junior residents were interested in skills-based programming to address physician burnout and were more amenable to wellness curricula as part of the mandatory didactic schedule. However, senior residents were more interested in professional identity development, including discussions about difficult clinical content, exploration of negative outcomes, ethical issues unique to psychiatry, and career planning. Professional identity development was consistently identified as essential to wellness, though making these discussions "mandatory" was controversial. Senior residents wanted flexibility to attend wellness offerings, or to find their own outlets for supporting wellbeing and professional identity formation.

One resident reflected:

"Although self-care is important...there is minimal [support around] difficult communication with patients, dealing with negative outcomes and countertransference reactions... It should be a mandatory part of the ... curriculum."

Another resident reflected:

"The elephant in the room in the topic of resilience... has always been time. It is only possible to foster wellness in residents, as with anyone else, through the availability of free time and autonomy."

Second, we identified that clear communication and community building are essential to wellness. Our needs assessment identified clear communication pathways and community building as a challenge in a large residency program. Residents noted that a feeling of anonymity and disconnection from leadership and decision-making processes can occur in a larger training program. Related to community-building, residents described a wish for more informal interactions with faculty.

One resident reflected,

“...creating opportunities for residents to relax and share stories with staff and colleagues."

Another resident stated:

"The culture of wellness within the faculty is likely to have a large impact on resident wellness and how residents develop coping skills. Including faculty in wellness offerings, either with residents or separate from residents, might be helpful."

Third, our analysis demonstrated that systems-level policies lay the groundwork for wellness. Residents resoundingly reported a greater impact from organizational/systematic supports in the form of policies and access to mental health resources than from isolated seminars. For example, residents advocated for clear policies that address barriers to taking personal leaves or sick days during training (including 
perceived stigma), as well as clear pathways for accessing mental health supports during residency.

One resident reflected:

"I think part of resident wellness is thinking about whether the system is actually conducive to wellness. Examples ... include allowing residents more flexibility in scheduling (although psych is better than most programs), reducing mandatory assignments and maximizing electives ... Adding wellness activities may be beneficial but not in a vacuum."

Another resident suggested:

"Ways to ensure ...confidentiality for residents when engaging in mental health services"

\section{Development of a Wellness Strategy}

We used our needs assessment results to inform the development of a wellness action plan, based on the priority areas identified in the needs assessment and made possible by the new subcommittee infrastructure. Embedded in our process was opportunity for yearly reflection and re-focus, with collaboration between our faculty and residents. See Table 1 for a description of programmatic initiatives, which were inspired by the guiding principles from our needs assessment, and structured based on the APA Wellbeing Ambassadors framework.

In response to the principle of "one size does not fit all," a wellness curriculum was offered to first year residents [14], while optional offerings, with a focus on professional development [15], were made available to senior residents (Table 1, Bullet 3). Our channels of communication were updated and opportunities for both formal and informal community building were established (Table 1, Bullets 1 and 4). Administratively, our program observed the strong impact that policy changes can have towards a culture of wellbeing. Since development of our wellness strategy, the RPC subcommittee supported the development of two new policies (Table 1, Bullets 6 and 7) -the Waiver of Training Policy, which per Canadian Royal College of Physicians and Surgeons allows residents who are meeting educational competencies to waive up to 12 weeks of training time from a prior medical/personal leave; and the Adverse Events Policy, which outlines standardized educational and supportive responses for residents who have experienced serious adverse patient events in training. Residents have reported that these changes in policy have greatly impacted their wellness.

We believe our scholarly process, subcommittee infrastructure, and wellness action plan may be helpful to other residencies embarking on developing a wellness initiative.
Our strategy ensured that the RPC Wellbeing Subcommittee was present at key administrative meetings and stayed accountable to program leadership by reporting back on successes, challenges, and deliverables. As culture change takes time, yearly revisiting of goals and priorities allowed for reflection on subtle and large changes to culture occurring since starting our wellness strategy. By using the APA Wellbeing Ambassador framework, we were able to focus our efforts, without ignoring important aspects of trainee wellness. We learned that our resident community valued opportunities for reflection and skill building (\#2 and 3), but was most engaged on topics related to community building (\#4), access to mental health care and supports (\#5), and improvements to the workplace environment leading to transformed institutional culture (\#6 and 7). Psychiatry residents described being knowledgeable about the risk of burnout, and were less interested in having more education about types of physician distress. Therefore, our action plan did not include additional lectures about these topics. Instead, this information was provided on our residency website (Table 1, Bullet 1), and we focused on later steps of the APA framework. In this way, we integrated the APA framework and the lessons learned from our needs assessment to form our strategy.

The terms of reference for the RPC subcommittee strongly relied on lessons learned from our needs assessment. The subcommittee's purpose is to elicit and understand issues affecting wellbeing in the residency, to facilitate development of programs that support connection, community, and resilience, and, especially, to foster development of policies in support of a culture of wellbeing in the residency. Over the 3 years since beginning our wellness strategy, it was beneficial to have the same faculty involved in articulating wellness initiatives yearly with the resident leads. The annual change in resident wellness leads allowed for diverse resident representation and awareness of evolving concerns. As a result, wellness initiatives were strongly guided by resident concerns, while ensuring institutional memory, RPC support, and adherence to our initially defined purpose. In this way, the importance of wellness and wellbeing initiatives were emphasized in residency program operations. As with most quality improvement (QI) initiatives, the change in culture observed in our program happened iteratively.

There were limitations to our process. Firstly, a small subset of residents participated in the Town Hall, which may represent selection bias for those residents invested in wellness initiatives. It is possible that our wellness strategy misses important wellness issues impacting residents who chose not to participate, and unfortunately due to privacy concerns, we did not gather additional demographics. Further, our needs assessment and wellness strategy attend to the identified needs of a single, large residency programone that has significant resources dedicated to planning and 
Table 1 Lessons learned from our needs assessment and alignment of our action plan with the APA Toolkit for Well-being Ambassadors

Lessons learned from our needs assessment:

- One size does not fit all, as residents have diverse needs depending on their stage of training

- Clear communication and community building are essential to wellness

- Systems-level policies lay the groundwork for wellness

With consideration of these principles, we aligned our interventions with the APA Wellbeing Ambassador Framework

\section{APA framework}

1. Educate and increase awareness

2. Designate time for reflection

3. Teach practical skills

4. Build community

5. Ensure access to care

6. Improve workplace environment

7. Transform institutional culture
Action plan and interventions made

Updates to our residency website to include resources available to trainees:

1. Information about burnout and wellness

2. Channels to access local, institutional, and regional supports

3. Resident-developed infographic about maintaining wellbeing through the COVID-19 pandemic

Acknowledge the tension between opportunities for reflection and added demands on trainee's time

1. Evidence-informed wellness curriculum [14] for first year residents, emphasizing reflection and skill development before the start of clinical rotations

2. Optional Professionalism seminar [15] for senior residents: "Salon style" and hosted in faculty and resident homes (pre-pandemic), facilitating opportunities for faculty and residents to discuss controversial topics in the field that influence identity formation as psychiatrist and physician

3. Upreach Mentorship: Informal opt-in group mentorship opportunities with faculty and resident peers

4. Through the COVID-19 pandemic, regular emails from Program Director to residents, which shared different wellbeing resources, including reflection and mindfulness

1. Resident-led social event planning, emphasizing opportunities for dry social events and shared activities

2. Resident retreats with protected time

3. Residency-wide efforts to improve communication strategy of postgraduate leadership, emphasizing transparency, diverse modes of communication (email, in-person, virtual drop-in office hours, communication through site-based chief residents and site directors)

4. Weekly resident lunches, continued virtually through the COVID-19 pandemic with reimbursement for lunch

1. Resident Advisor: Psychiatry faculty member who provides confidential discussion, advice, or involvement, all at an arm's length from the residency program. The Resident Advisor can help residents navigate challenges in the system, or access care

2. Postgraduate Wellness Office: Offers a broad range of mental health supports and navigates personal or medical leaves and accommodations

3. Faculty Wellness Lead: Acts as liaison to centralized Postgraduate Wellness Committee of Postgraduate Wellness Office, which includes representation from other residency programs, and allows for up-to-date information transfer to Residency Program Committee (RPC) and residents

Development of formal RPC policies that support resident wellness, created in collaboration with residents including:

- Adverse Events Policy

- Waiver of Training Policy operations across multiple academic and community sites. We believe that the APA Toolkit [11] used to guide our QI process is easily tailored to individual program needs as it prompts programs to take inventory of its strengths and weaknesses, including suggestions for how to advocate for organizational resources in support of wellness.

Finally, wellness initiatives do not occur in a vacuum. In Canada, Competency-Based Medical Education (CBME) [16] implementation has presented new challenges to educators and residents alike. The data from our needs assessment was collected before CBME implementation. Residency is a time of steep growth and professional identity formation [17] and changes to curricular infrastructure, assessment, and environmental challenges can add new stress and uncertainty for residents, especially in the early years of changes. As a result, our residency program will pursue a renewed needs assessment with a similar constant comparison process to re-evaluate the goals and priorities established 3 years ago, to better understand how our learner's wellness needs have evolved 
and to continue to actively respond to these needs using the APA framework.

Acknowledgements The authors would like to thank prior Resident Wellness Committee leads, Drs. Shale Farber and Alvin Keng, and current leads Drs. Erene Stergiopoulos and Nikhita Singhal. We would also like to thank members of PRPC Subcommittee on Wellbeing at the University of Toronto. We would like to thank Dr. Richard Summers and the members of the APA's Committee on Psychiatrist Wellbeing and Burnout, who developed the APA Toolkit for Wellbeing Ambassadors framework adopted for this initiative.

\section{Declarations}

Ethics Approval Our study, which includes Quality Improvement data from an educational needs assessment, has been exempt from institutional review board review at the University of Toronto Human Research Ethics Board.

Disclosures On behalf of all authors, the corresponding author states that there is no conflict of interest.

\section{References}

1. Jennings ML, Slavin SJ. Resident wellness matters: optimizing resident education and wellness through the learning environment. Acad Med. 2015;90:1246-50.

2. Mata DA, Ramos MA, Bansal N, Khan R, Guille C, Di Angelantonio E, et al. Prevalence of depression and depressive symptoms among resident physicians: a systematic review and meta-analysis. JAMA. 2015;314:2373-83.

3. West CP, Huschka MM, Novotny PJ, Sloan JA, Kolars JC, Habermann TM, et al. Association of perceived medical errors with resident distress and empathy: a prospective longitudinal study. JAMA. 2006;296:1071-8.

4. Fahrenkopf AM, Sectish TC, Barger LK, Sharek PJ, Lewin D, Chiang VW, et al. Rates of medication errors among depressed and burnt out residents: prospective cohort study. BMJ. 2008;336:488-91.

5. Benson NM, Chaukos D, Vestal H, Chad-Friedman EF, Denninger JW, Borba CPC. A qualitative analysis of stress and relaxation themes contributing to burnout in first-year psychiatry and medicine residents. Acad Psychiatry. 2018;42:630-5.

6. Dyrbye LN, West CP, Satele D, Boone S, Tan L, Sloan J, et al. Burnout among U.S. medical students, residents, and early career physicians relative to the general U.S. population. Acad Med. 2014;89:443-51.
7. Ey S, Moffit MJ, Kinzie M, Brunett PH. Feasibility of a comprehensive wellness and suicide prevention program: a decade of caring for physicians in training and practice. J Grad Med Educ. 2016;8(5):747-53.

8. Ey S, Moffit M, Kinzie JM, Choi D, Girard DE. "If you build it, they will come': attitudes of medical residents and fellows about seeking services in a resident wellness program. J Grad Med Educ. 2013;5(3):486-92.

9. Panagioti M, Panagopoulou E, Bower P, Lewith G, Kontopantelis $\mathrm{E}$, Chew-Graham $\mathrm{C}$, et al. Controlled interventions to reduce burnout in physicians: a systematic review and meta-analysis. JAMA Intern Med. 2017;177:195-205.

10. Chaukos D, Vestal HS, Bernstein CA, Belitsky R, Cohen MJ, Hutner $\mathrm{L}$, et al. An ounce of prevention: a public health approach to improving physician well-being. Acad Psychiatry. 2018;42:150-4.

11. Goldman ML, Bernstein C, Chilton J, Aggarwal R. APA Toolkit for Well-being Ambassadors. In: Well-being Resources. American Psychiatric Association. 2017. https://www.psychiatry.org/ File\%20Library/Psychiatrists/Practice/Well-being-and-Burnout/ APA-Well-being-Ambassador-Toolkit-Manual.pdf. Accessed 8 Jan 2018.

12. Lohr K, Weinhardt M, Sieber S. The World Café as a participatory method for collecting qualitative data. Int J Qual Meth. 2020;19:1-15.

13. Lillemor R-M. Hallberg (2006) The "core category" of grounded theory: Making constant comparisons. International Journal of Qualitative Studies on Health and Well-being 1:3:141-148. https://doi.org/10.1080/17482620600858399

14. Chaukos D, Chad-Friedman E, Mehta DH, Byerly L, Celik A, McCoy TH, et al. SMART-R: a prospective cohort study of a resilience curriculum for residents by residents. Acad Psychiatry. 2018;42:78-83.

15. Freudenreich O, Kontos N. "Professionalism, Physicianhood, and Psychiatric Practice": Conceptualizing and implementing a senior psychiatry resident seminar in reflective and inspired doctoring. Psychosomatics. 2019;60:246-54.

16. Harris KA, Nousiainen MT, Reznick R. Competency-based resident education-the Canadian perspective. Surgery. 2020;167:681-4.

17. Cruess RL, Cruess SR, Boudreau DJ, Snell L, Steinert Y. A schematic representation of the professional identity formation and socialization of medical students and resident a guide for medical educators. Acad Med. 2015;90:718-29.

Publisher's Note Springer Nature remains neutral with regard to jurisdictional claims in published maps and institutional affiliations. 